\title{
Inhibitory Effects of Adlay Extract on Melanin Production and Cellular Oxygen Stress in B16F10 Melanoma Cells
}

\author{
Huey-Chun Huang ${ }^{1}$, Wan-Yu Hsieh ${ }^{2}$, Yu-Lin Niu ${ }^{3}$ and Tsong-Min Chang ${ }^{2, *}$
}

1 Department of Medical Laboratory Science and Biotechnology, China Medical University, No. 91 Hsueh-Shih Road, Taichung 40402, Taiwan; E-Mail: lchuang@mail.cmu.edu.tw

2 Department of Applied Cosmetology \& Master Program of Cosmetic Sciences, Hungkuang University, No. 1018, Sec. 6, Taiwan Boulevard, Shalu Dist., Taichung City 43302, Taiwan; E-Mail: opency.tw@gmail.com

3 Niuer International Skincare Science Research Institute, 7F, No. 618, Ruiguang Rd., Neihu Dist., Taipei 114, Taiwan; E-Mail: niuer80@gmail.com

* Author to whom correspondence should be addressed; E-Mail: ctm@sunrise.hk.edu.tw; Tel.: +886-4-2631-8652 (ext. 2216); Fax: +886-4-2631-5843.

Received: 6 January 2014; in revised form: 12 September 2014 / Accepted: 15 September 2014 / Published: 19 September 2014

\begin{abstract}
The aim of this study was to determine the effects of adlay extract on melanin production and the antioxidant characteristics of the extract. The seeds were extracted by the supercritical fluid $\mathrm{CO}_{2}$ extraction (SFE) method. The effect of adlay extract on melanin production was evaluated using mushroom tyrosinase activity assay, intracellular tyrosinase activity, antioxidant properties and melanin content. Those assays were performed spectrophotometrically. In addition, the expression of melanogenesis-related proteins was determined by western blotting. The results revealed that the adlay extract suppressed intracellular tyrosinase activity and decreased the amount of melanin in B16F10 cells. The adlay extract decreased the expression of microphthalmia-associated transcription factor (MITF), tyrosinase, tyrosinase related protein-1 (TRP-1) and tyrosinase related protein-2 (TRP-2). The extract also exhibited antioxidant characteristics such as free radical scavenging capacity and reducing power. It effectively decreased intracellular reactive oxygen species (ROS) levels in B16F10 cells. We concluded that the adlay extract inhibits melanin production by down-regulation of MITF, tyrosinase, TRP-1 and TRP-2. The antioxidant properties of the extract may also contribute to the inhibition of melanogenesis. The adlay extract can therefore be applied as an inhibitor of melanogenesis and could also act as a natural antioxidant in skin care products.
\end{abstract}


Keywords: adlay; MITF; tyrosinase; melanin; ROS

\section{Introduction}

Melanin is a pigment produced by epidermal melanocytes and is responsible for skin color and for protecting skin from environmental UV damage. In the melanin biosynthesis pathway, tyrosinase catalyzes the rate-limiting steps in which L-tyrosine is hydroxylated to L-3,4-dihydroxyphenylalanine (L-DOPA), and L-DOPA is further oxidized into the corresponding $o$-quinone. Thus, tyrosinase is a major target in screening inhibitors for melanin synthesis. Several skin depigmenting chemicals such as kojic acid [1] and arbutin [2], which act as tyrosinase inhibitors, have been applied in skin whitening products for the treatment or prevention of abnormal skin pigmentation [3]. It has been reported that microphthalmia-associated transcription factor (MITF), tyrosinase related protein-1 (TRP-1) and tyrosinase related protein-2 (TRP-2) also contribute to the production of melanin [4,5]. Additionally, it has been reported that melanogenesis produces hydrogen peroxide $\left(\mathrm{H}_{2} \mathrm{O}_{2}\right)$ and other reactive oxygen species (ROS) that expose the human melanocytes to high levels of oxidative stress [6]. ROS have been shown to play a significant role in the regulation of melanin synthesis, whereas ROS scavengers and inhibitors of ROS generation may down-regulate UV-induced human melanogenesis [7]. The contribution of ROS to melanogenesis has been studied using antioxidants such as $N$-acetyl cysteine to abolish UVB-induced $\alpha$-melanocyte stimulating hormone [8]. Stimulation of an endogenous antioxidant, metallothionein, also suppresses melanogenesis in melanocytes [9]. Furthermore, it is reported that UV light radiation causes the synthesis of ROS in skin [10]. Therefore, the use of antioxidants to protect human skin from the harmful effects of UV radiation is a new trend that has attracted increasing interest in the fields of dermatology and skin care products in recent years [11]. The B16F10 murine melanoma cell line was reported to be a good model for studying human melanoma [12]. Hence, we chose this cell line in the present study to evaluate a natural inhibitor of melanogenesis.

Adlay (Coix lachryma-jobi L. var. ma-yuen Stapf, Job's tears) is a traditional Chinese medicinal plant that has been reported to show various pharmacological activities, such as anti-inflammatory [13] and anti-allergic effects [14]. Furthermore, the methanolic extract of adlay seeds inhibits nitric oxide (NO) and $\mathrm{O}^{-\bullet}$ production by activated macrophages [15]. In addition, the adlay seeds' methanolic extracts exhibited antioxidant activities [16]. However, to date there are no scientific reports on dermatological applications of the seed extract of this plant. The aim of this study is to investigate the inhibitory effect of supercritical fluid $\mathrm{CO}_{2}$ extract of adlay seeds on melanogenesis in $\mathrm{B} 16 \mathrm{~F} 10$ melanoma cells and evaluate the potential antioxidant characteristics of the extract.

\section{Results and Discussion}

\subsection{Effects of Adlay Extract on Mushroom Tyrosinase Activity and Melanogenesis}

To determine the potential inhibitory effect of adlay extract on mushroom tyrosinase activity, enzyme inhibition experiments were carried out in triplicate. Kojic acid was used as a positive standard. The results indicated that mushroom tyrosinase activity was inhibited by various concentrations of adlay extract $(25-250 \mathrm{mg} / \mathrm{mL})$. The residual tyrosinase activity was $69.82 \% \pm 6.29 \%, 63.57 \% \pm 3.66 \%$, 
$46.68 \% \pm 3.52 \%$ and $38.85 \% \pm 3.31 \%$ of the control for $25,50,125$ and $250 \mathrm{mg} / \mathrm{mL}$ of adlay extract, respectively. Simultaneously, the tyrosinase activity was inhibited by kojic acid and the remained enzyme activity was $45.67 \% \pm 4.21 \%$ of positive control. Mushroom tyrosinase is routinely used for screening potential inhibitors of melanogenesis in vitro experiments. Increasing concentration of extract in the reaction mixture exhibited increased inhibitory activity of the enzyme. At $250 \mathrm{mg} / \mathrm{mL}$ levels, as much as $60 \%$ activity of the enzyme was inhibited. In contrast, kojic acid a well-known inhibitor of mushroom tyrosinase showed an inhibition of about $50 \%$ at $200 \mu \mathrm{M}$ concentrations $(0.028 \mathrm{mg} / \mathrm{mL})$ (Figure 1A). Thus, the adlay extract seems to exhibit potent inhibition of mushroom tyrosinase activity.

We then studied the effect of adlay extract on the production of melanin in B16 melanoma cells. Figure 1B shows the results of this study. For comparison, the effect of arbutin, a skin-lightening compound on the melanin production in this cell line at $2 \mathrm{mM}$ concentration $(0.545 \mathrm{mg} / \mathrm{mL})$ is given. As is evident, increased concentrations of adlay extract inhibited the melanin production increasingly with an $\mathrm{IC}_{50}$ value of $61.21 \mathrm{mg} / \mathrm{mL}$. Thus adlay extract seems to be efficient in inhibiting melanin production in the B16 melanoma cell line.

In order to see if the above inhibition is caused by the inhibition of endogenous tyrosinase activity, intracellular tyrosinase activity was determined after treating the cells with various concentrations of the adlay extract. Again, increasing concentrations of adlay extract caused increased inhibition of intracellular tyrosinase activity with an $\mathrm{IC}_{50}$ value of $36.31 \mathrm{mg} / \mathrm{mL}$ (Figure 1C). Thus, the results presented in Figure 1 clearly indicate that adlay extracts not only possess potent inhibition of tyrosinase activity, but also have significant ability to reduce the melanin production in cultured cells.

One might argue that the reduced melanin production and reduced intracellular tyrosinase activity observed with B16 melanoma cells could be due to the cytotoxicity of the adlay extracts, which might have killed the cells and reduced the viable cells there by showing reduced melanin production and reduced enzyme activity. To assess this possibility, the viability of the adlay extract treated cells were determined by MTT assay. MTT is a pale yellow compound that is converted by living cells to a dark blue formazan product, in contrast, dead cells do not perform this reaction and hence remain yellow colored. Therefore, living cells will appear blue while dead cells appear yellow after treatment with MTT. Experiments conducted with MTT treatment on B16 melanoma cells with various concentrations of the SFE $(20,30$ and $40 \mathrm{mg} / \mathrm{mL})$ for $24 \mathrm{~h}$ did not show any significant difference in the viability of the cells thereby indicating that the adlay extract is not cytotoxic to B16F10 cells (data not shown). In the experiments described here, alpha-melanocyte-stimulating hormone ( $\alpha-\mathrm{MSH}$ ) was used as a cAMP inducer to stimulate melanin synthesis. It is reported that $\alpha$-MSH can bind melanocortin 1 receptor (MC1R) and activate adenylate cyclase, which in turn catalyzes ATP to cAMP and increases intracellular cAMP levels [17]. The results showed that the adlay extract inhibited melanogenesis induced by $\alpha$-MSH-mediated intracellular cAMP up-regulation. Furthermore, some chemical depigmenting agents show side effects such as the genotoxic effect of arbutin [18] and pigmented contact dermatitis due to kojic acid [19]. Hence, searching for a safe and effective skin whitening agent remains a goal in the fields of cosmetic research and development. 
Figure 1. The inhibitory effects of adlay extract on melanogenesis. (A) The effects of adlay extract on mushroom tyrosinase activity; (B) The effects of adlay extract on melanin content in B16F10 cells; (C) The effects of adlay extract on tyrosinase activity in B16F10 cells. The results are presented as percentages of the control, and the data are presented as the mean \pm S.D. of three separate experiments. Black bars and lines on each bar indicate the mean and standard deviation. The values are significantly different compared with the control. * $p<0.05, * * p<0.01$.

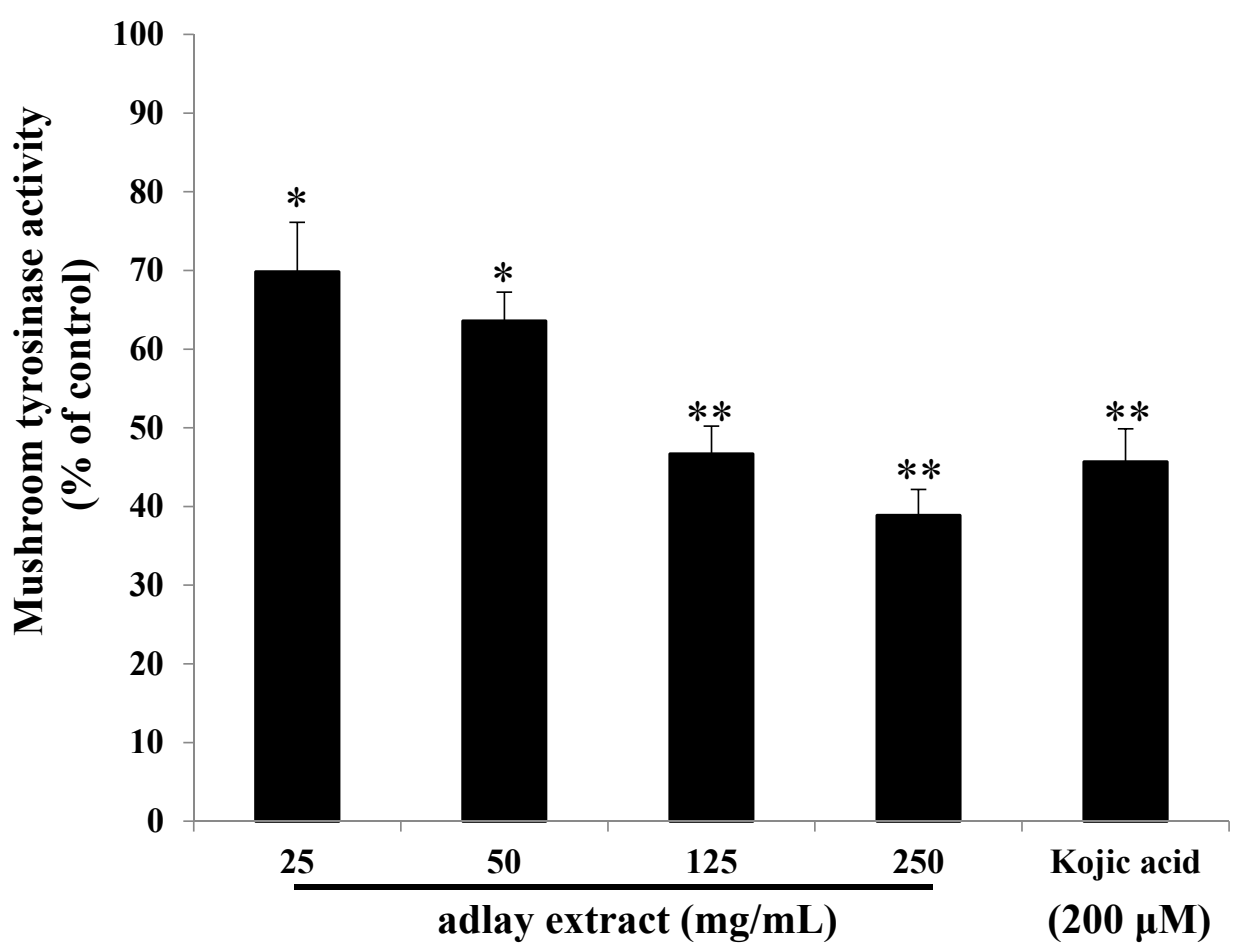

(A)

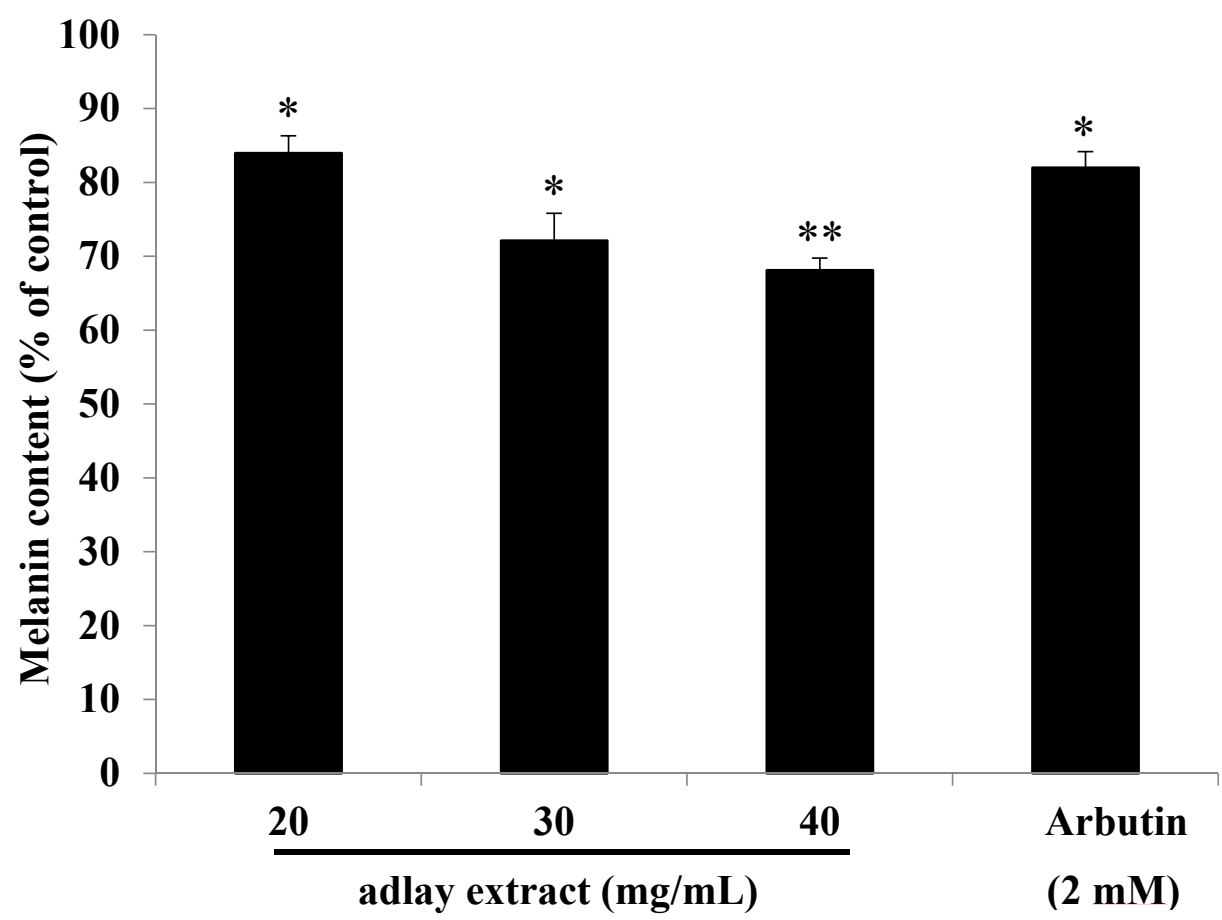

(B) 
Figure 1. Cont.

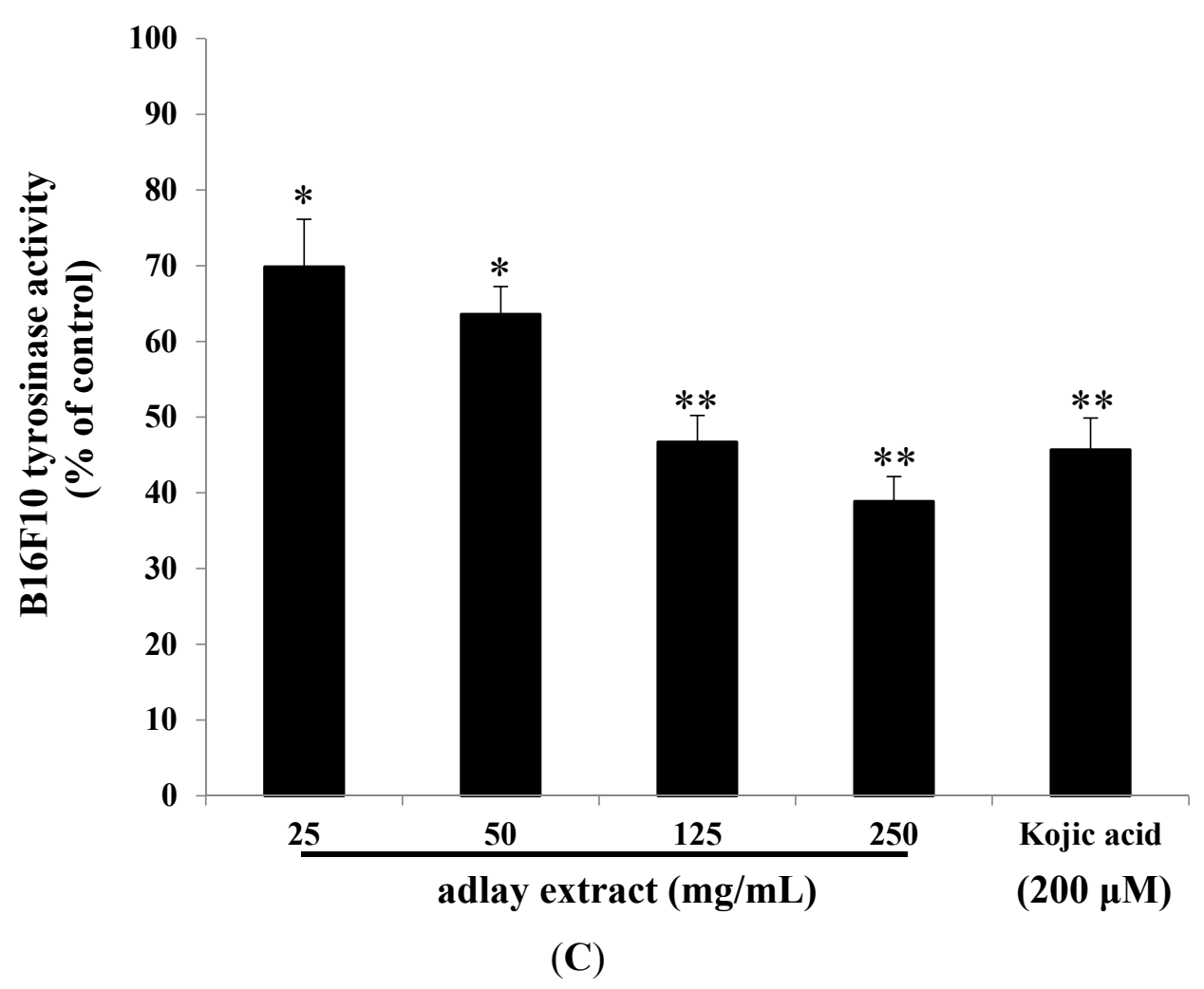

To further confirm these findings and to assess the effect of adlay extracts on the activity of other melanogenic enzymes, we conducted the following studies. It is now well established that apart from tyrosinase, a number of other proteins also participate in melanogenesis. TRP-1, which converts dopachrome to 5,6-dihydroxyindole-2-carboxylic acid and TRP-2, which oxidizes 5,6-dihydroxyindole-2-carboxylic acid, are two other proteins that are associated with melanogenesis. MITF is another protein that plays a crucial role in melanogenesis by being a major transcriptional regulator of tyrosinase, TRP-1 and TRP-2 genes in animals. Therefore, we tested the levels of these proteins by western blot analysis after expose to adlay extracts.

Figure 2A shows the western blot analysis of tyrosinase, TRP-1, TRP-2 and MITF along with a control protein GAPDH after treatment with adlay extracts and kojic acid. As expected, different treatments did not affect the levels of GAPDH. The kojic acid, a potent inhibitor of tyrosinase also did not affect the expression levels of tyrosinase, TRP-1, TRP-2 and MITF. However, different concentrations of adlay extract affected the expression levels of these proteins.

To assess the effect of adlay extracts on the cellular melanin production, we determined the mRNA levels of MITF in cells treated with $\alpha$-MSH and adlay extracts. The $\alpha-\mathrm{MSH}$ treated cells showed increased levels of MITF as expected. Interestingly adlay extract treated cells showed reduction in the expression of MITF mRNA levels (Figure 2B). 
Figure 2. The effects of adlay extract on melanogenesis-related protein expression levels. (A) Western blotting of cellular proteins in B16F10 cells; (B) Total RNA from B16F10 cells treated with adlay extract $(40 \mathrm{mg} / \mathrm{mL})$ collected at the indicated time. microphthalmia-associated transcription factor (MITF) mRNA levels were examined by real time RT-PCR, using glyceraldehyde 3-phosphate dehydrogenase (GAPDH) as an internal control. $(* p<0.05$ vs. control).

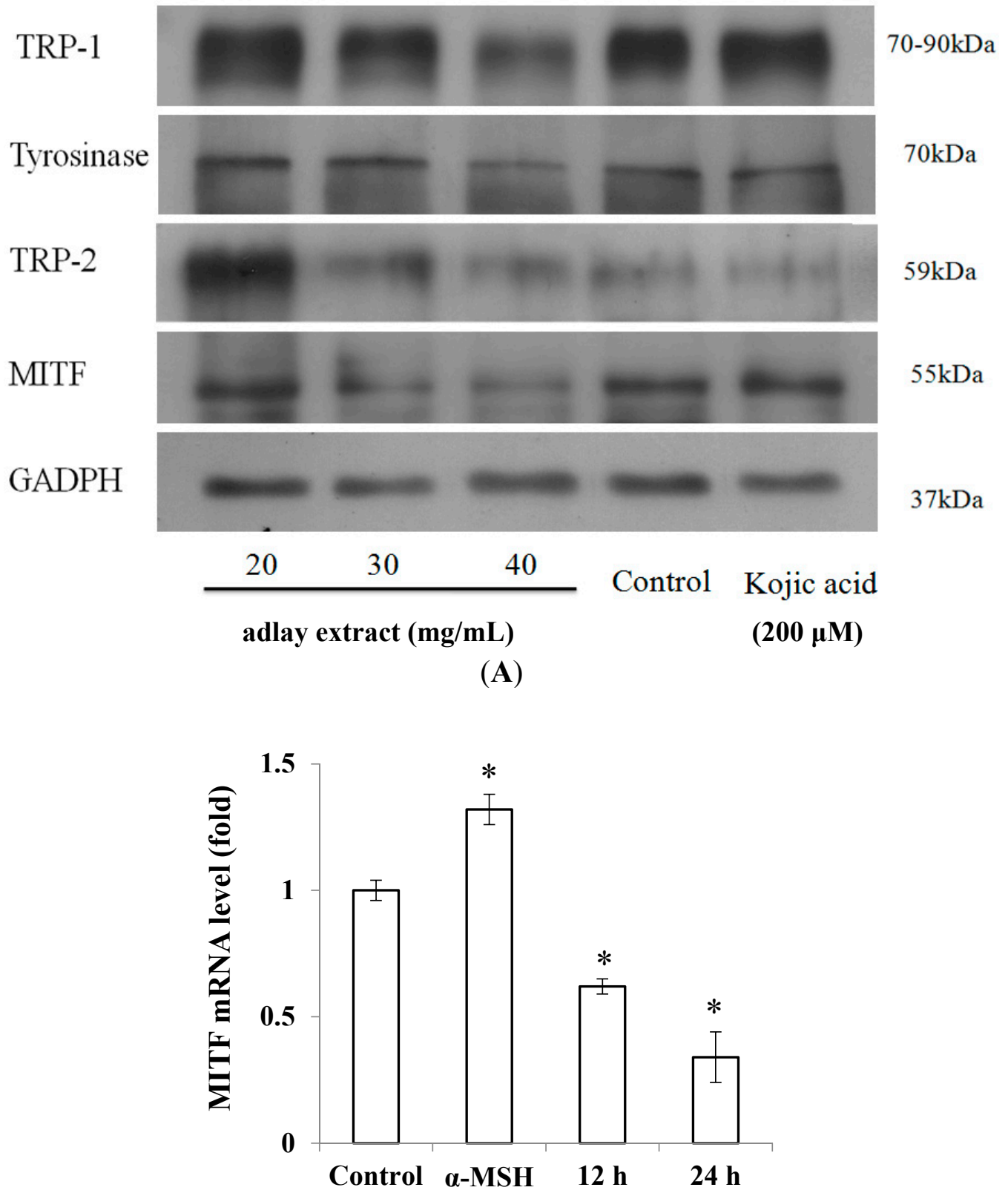

(B) 


\subsection{Antioxidant Characteristics of the Adlay Extract}

We then focused out attention on the antioxidant properties of adlay extracts. To this end, we carried out the radical scavenging assay using 2,2-diphenyl-1-picrylhydrazyl (DPPH). DPPH is a stable free radical that can interact with different antioxidant molecules. During the process, it gets reduced to a colorless compound. Therefore, antioxidant properties of many compounds can be easily determined by their ability to reduce this colored compound by monitoring the decrease in absorbance at $517 \mathrm{~nm}$ using a UV-visible spectrophotometer. For control, we used vitamin C, vitamin $\mathrm{E}$ and butylated hydroxyanisole (BHA). The results shown in Figure 3A clearly indicate that adlay extracts possess antioxidant properties.

The $\mathrm{ABTS}^{+}$free radical was used to determine the free radical scavenging activity of the adlay extract. Interaction of antioxidants with $\mathrm{ABTS}^{+}$transfer hydrogen atoms to $\mathrm{ABTS}^{+}$thus neutralizing its free radical character. The adlay extract showed similar $\mathrm{ABTS}^{+}$free radical scavenging activities as that of Vitamin C or BHA (Figure 3B). As shown in Figure 3C, adlay extracts at higher concentration exhibited the same reducing capacity as that of BHA $(25 \mu \mathrm{g} / \mathrm{mL})$. The antioxidant activity of phenolic compounds is probably due to their redox properties, which can play an important role in adsorbing and neutralizing free radicals, quenching singlet and triplet oxygen, or decomposing peroxides. At lower concentration range $(5-125 \mathrm{mg} / \mathrm{mL})$, increasing concentration of extract shows a proportional increase in phenolic content. It was found that higher concentrations of the extract $(125$ and $250 \mathrm{mg} / \mathrm{mL})$ show similar phenolic content as that of gallic acid (Figure 3D). This is probably due to some bioactive compounds such as polyphenols including tannins and flavonoid existed in the extracts.

Therefore, we studied the effect of adlay extract on intracellular ROS levels. The study was conducted with the use of DCFH-DA (2',7'-dichlorofluorescein diacetate), which diffuses through the cell membrane easily and is hydrolyzed by the endogenous esterases to DCFH. DCFH thus formed reacts with ROS such as $\mathrm{H}_{2} \mathrm{O}_{2}$ to generate DCF. To confirm the antioxidant capacity of adlay extract in a cellular model, evaluation of intracellular ROS levels was done. Rapid increases in DCF indicate oxidation of DCFH by intracellular radicals (Figure 3E).

When exposed to UV light or environmental oxidizing agents, human skin experiences oxidative stress. It has been reported that ultraviolet irradiation induces the formation of ROS in cutaneous tissue [20], provoking damage such as enzyme inactivation and lipid peroxidation. The search for antioxidants with skin-depigmenting capabilities is driven by the hypothesis that oxidative stress resulting from UV-irradiation may contribute to the stimulation of melanogenesis. In addition, redox agents may also influence melanin production by interacting with the copper ion at the active site of tyrosinase or with $o$-quinones to block the oxidative polymerization of melanin intermediates. Interestingly, plant extracts such as chestnut flower extract [21] and Paeonia suffruticosa [22] have been reported to show antioxidant and antimelanogenic properties. Our research indicates that the apparent antioxidant capacity of adlay extract may contribute to its depigmenting activity and could be included in cosmetic formulations of skin care products. Certainly, the efficacy of adlay extract-containing products should be evaluated by applying to human study in the near future. 
Figure 3. The antioxidant characteristics of adlay extract. (A) DPPH scavenging capacity assay; (B) ABTS $^{+}$radical scavenging activity assay; (C) Determination of reducing capacity; (D) Measurement of total phenolic content; (E) ROS level assay. Different concentrations of the adlay extract and positive standards were used in the above assays. The results are expressed as percentages of the control. The data are presented as the mean \pm S.D. $* p<0.05, * * p<0.01$.

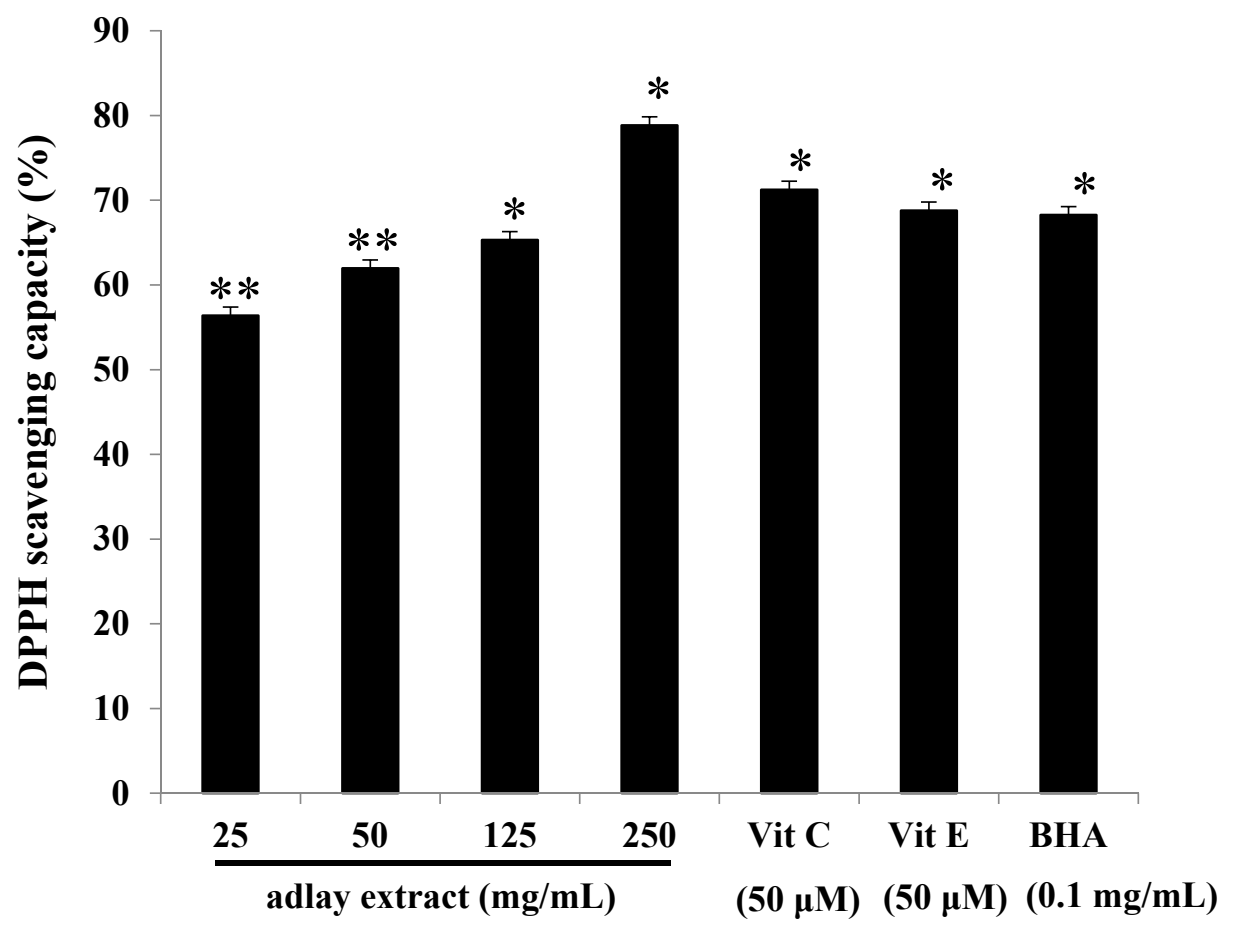

(A)

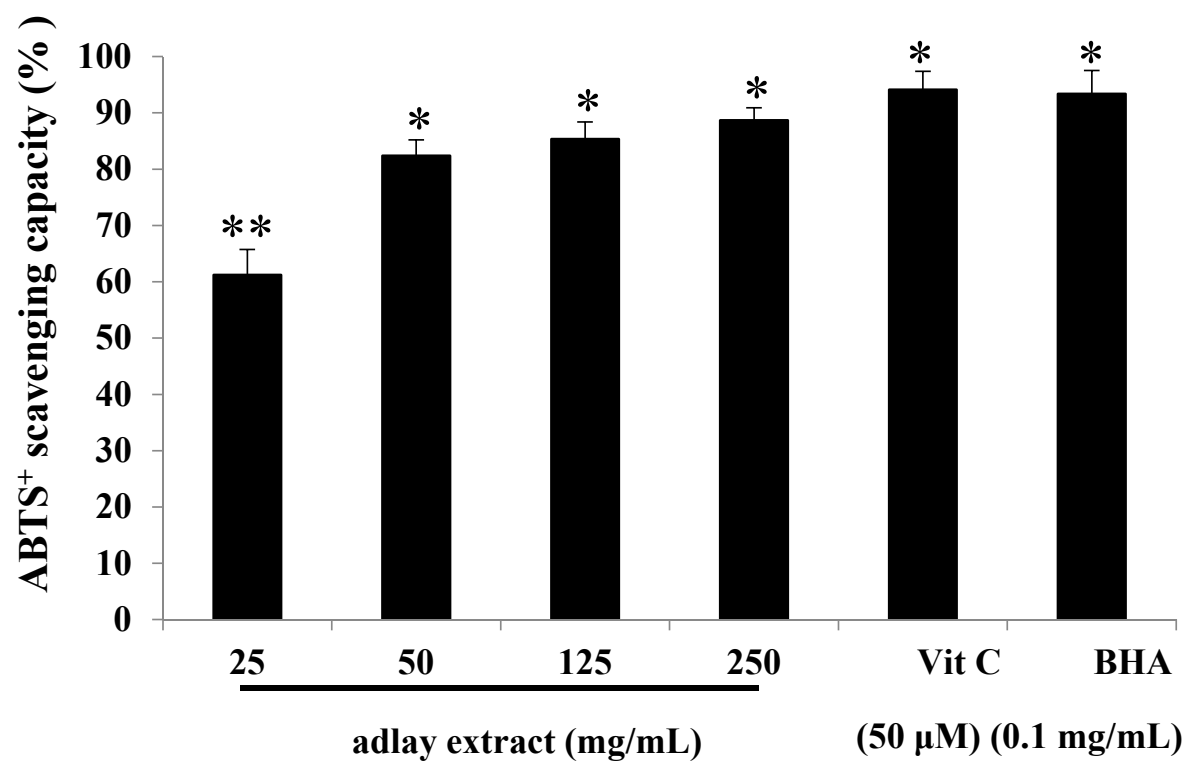

(B) 
Figure 3. Cont.

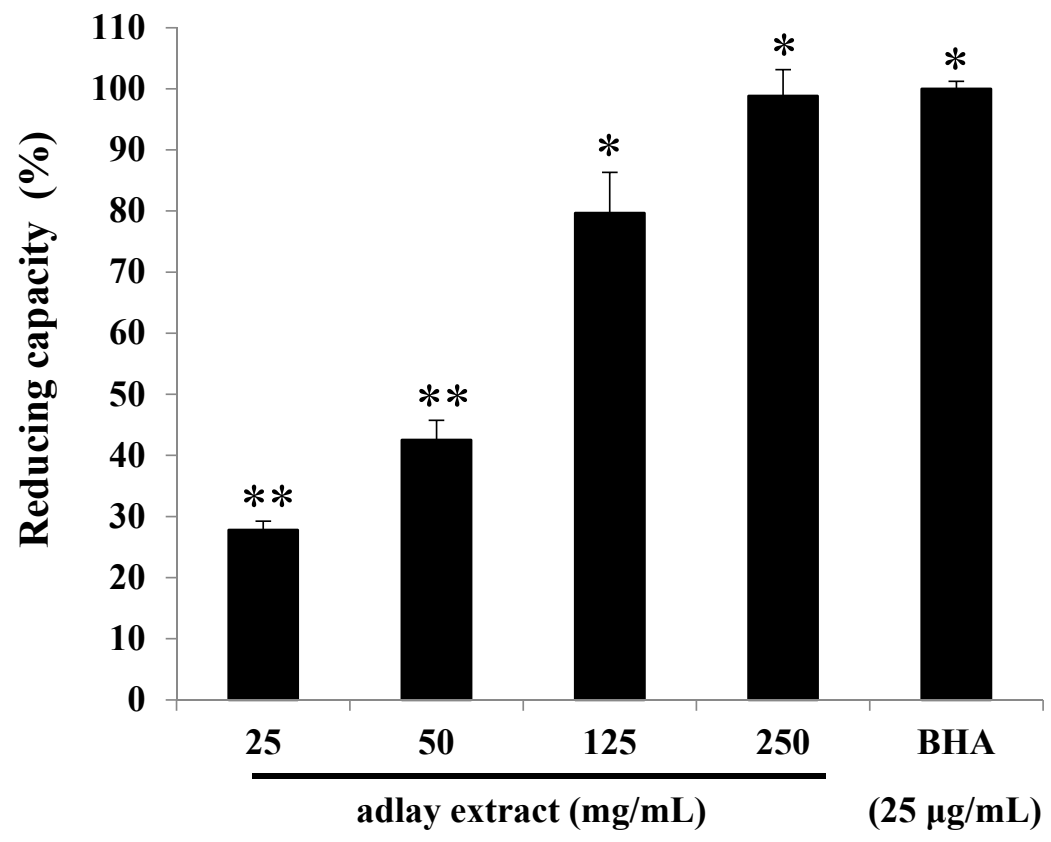

(C)

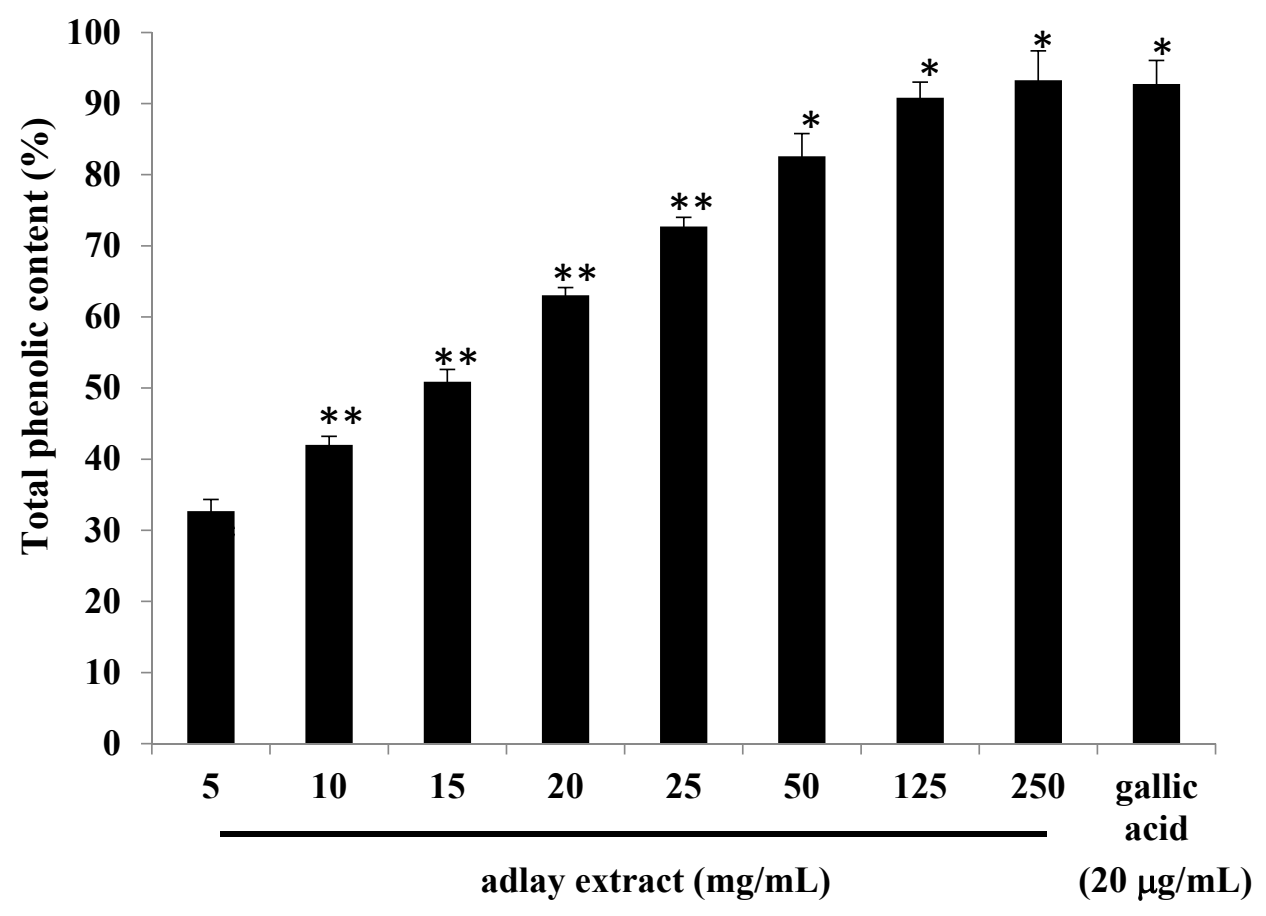

(D) 
Figure 3. Cont.

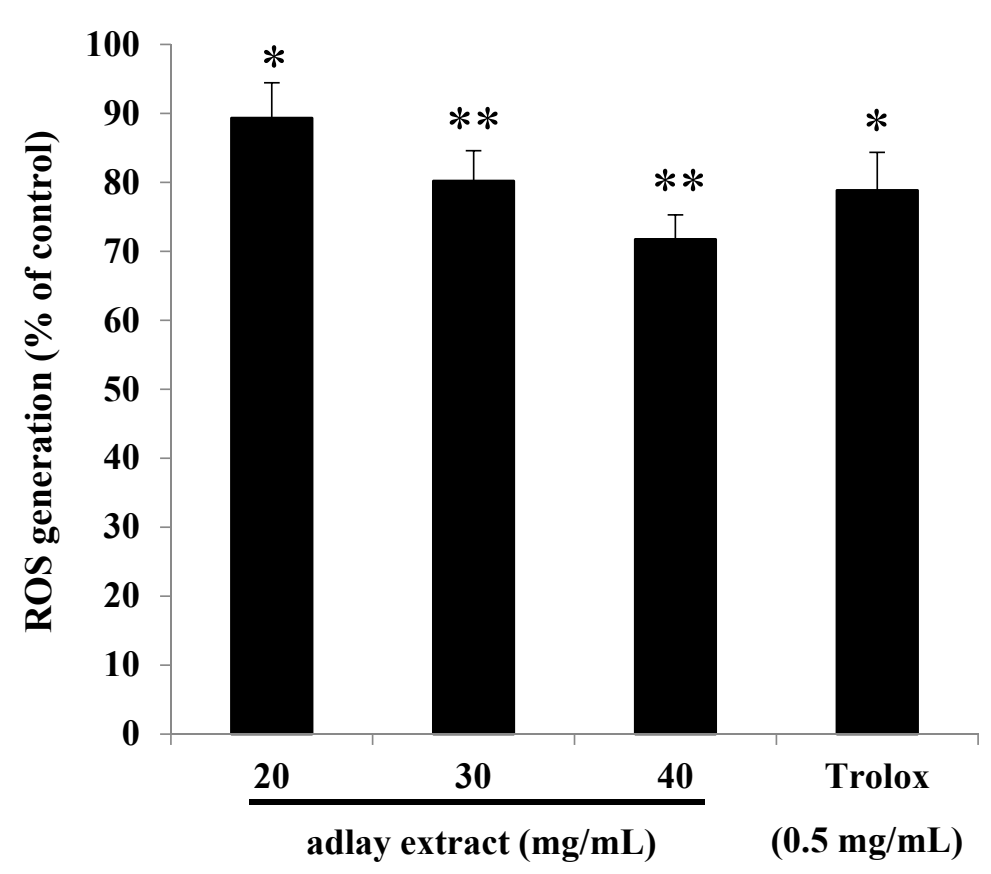

(E)

\section{Experimental Section}

\subsection{Chemicals and Reagents}

All chemical reagents were purchased from Sigma Chemical Co. (St. Louis, MO, USA). The antibodies were obtained from Santa Cruz Biotech (Santa Cruz, CA, USA), and the ECL reagent was from Millipore (Milford, MA, USA).

\subsection{Supercritical Fluid $\mathrm{CO}_{2}$ Extraction (SFE) of Adlay Seeds}

The adlay seeds were harvested in May 2012 from Nantou County, Taiwan. The seeds were washed, exposed to sunlight and air-dried for one day. The seeds were then dried at $80{ }^{\circ} \mathrm{C}$ for $2 \mathrm{~h}$ in an oven. The dried seeds were pulverized to a fine powder with a committed mill (Retsch Ultra Centrifugal Mill and Sieving Machine, Type ZM1, Haan, Germany). The adlay seed powder (75 g) was placed in the extraction vessel $(200 \mathrm{~mL})$ of the supercritical fluid $\mathrm{CO}_{2}$ extraction apparatus (SFE-400S-2000, Metal Industries Research and Development Centre; MIRDC; Kaohsiung, Taiwan). The extraction was performed with $10 \%$ co-solvent of ethanol in supercritical fluid $\mathrm{CO}_{2}$ (flow rate, $5.0 \mathrm{~mL} / \mathrm{min})$ at $5000 \mathrm{psi}(350 \mathrm{bar})$ at a temperature of $50{ }^{\circ} \mathrm{C}$ for $2 \mathrm{~h}$. The extracts were dried on a rotary evaporator at $40{ }^{\circ} \mathrm{C}$ under reduced pressure. The concentrated SFEs were weighed and stored at $-20{ }^{\circ} \mathrm{C}$. In the following experiments, the SFEs were re-dissolved in dimethyl sulfoxide (DMSO), as indicated, and the final concentrations of DMSO in the cellular experiments were less than $1 \%$. 


\subsection{Inhibitory Effects of Adlay Extract on Melanogenesis}

Inhibition assays of mushroom tyrosinase activities were conducted as previously described [23]. A reaction mixture containing 200 units (in $10 \mu \mathrm{L}$ ) of mushroom tyrosinase and $5 \mathrm{mM}$ DOPA in $50 \mathrm{mM}$ sodium phosphate buffer $\mathrm{pH} 6.8$ was incubated in 96 well microtiter plate at $37{ }^{\circ} \mathrm{C}$ for $30 \mathrm{~min}$. The reaction was started by the addition of enzyme. Following incubation, the amount of dopachrome produced in the reaction mixture was determined spectrophotometrically at $490 \mathrm{~nm}\left(\mathrm{OD}_{490}\right)$ using a microplate reader. The inhibition percentage at three doses for each experiment was calculated by the following equation: inhibition percentage of tyrosinase activity $(\%)=(B-A) \div A \times 100$, where $B$ is the mean of the measured OD 490 values of the blank control, and $A$ is the mean of the measured $\mathrm{OD}_{490}$ values for the adlay extract treated group.

The B16F10 cells (ATCC CRL-6475, BCRC60031) were obtained from the Bioresource Collection and Research Center (BCRC), Taiwan. The cells were maintained in DMEM (Hyclone, Logan, UT, USA) supplemented with $10 \%$ fetal bovine serum and $1 \%$ antibiotics at $37{ }^{\circ} \mathrm{C}, 5 \% \mathrm{CO}_{2}$ in a humidified incubator. The melanin content was measured as previously described by Tsuboi et al. [24], while the cellular tyrosinase activity was determined as described in an earlier publication with some modifications [25]. The cells were pretreated with $\alpha$-MSH $(100 \mathrm{nM})$ for $24 \mathrm{~h}$ and treated with either the adlay extract or arbutin for a further $24 \mathrm{~h}$, then the melanin content or intracellular tyrosinase activity was measured. After treatment, the cells were detached by incubation in trypsin/ethylenediaminetetraacetic acid (EDTA). After precipitation at 900 r.p.m for 5 min, cell pellets containing a known number of cells were solubilized in $1 \mathrm{~N} \mathrm{NaOH}$ at $60{ }^{\circ} \mathrm{C}$ for $60 \mathrm{~min}$. The melanin content was determined by spectrophotometric analysis by measuring its absorbance of $405 \mathrm{~nm}$. The intracellular tyrosinase activity was determined as follows: Cell extracts $(100 \mu \mathrm{L})$ were mixed with freshly prepared L-DOPA solution $\left(0.1 \%\right.$ in phosphate-buffered saline) and incubated at $37{ }^{\circ} \mathrm{C}$. The absorbance of this solution at $490 \mathrm{~nm}$ was measured with microplate reader Gen $5^{\mathrm{TM}}$ (BIO-TEK Instrument, Vermont, VT, USA) to estimate the production of dopachrome. Corrections were made for auto-oxidation of L-DOPA.

The western blotting experiments were performed as outlined in our earlier study [26]. The relative amounts of MITF, tyrosinase, TRP-1 and TRP-2 compared with total glyceraldehyde 3-phosphate dehydrogenase (GAPDH) were calculated and analyzed with Multi Gauge 3.0 software (Fuji, Tokyo, Japan).

\subsection{RT-PCR}

RNA samples were reverse-transcribed for $120 \mathrm{~min}$ at $37{ }^{\circ} \mathrm{C}$ with High Capacity cDNA Reverse Transcription Kit according to the standard protocol of the supplier (Applied Biosystems, Foster City, CA, USA). Quantitative PCR was performed by as follows: 10 min at $95{ }^{\circ} \mathrm{C}, 40$ cycles of $15 \mathrm{~s}$ at $95^{\circ} \mathrm{C}$, 1 min at $60^{\circ} \mathrm{C}$ using $2 \times$ Power SYBR Green PCR Master Mix (Applied Biosystems, Foster City, CA, USA) and $200 \mathrm{nM}$ of forward and reverse primers (MITF gene forward primer: GGCCAAGGCAG AGCAACTT; MITF gene reverse primer: GCCCATGGTGGCAAGCT; GAPDH gene forward primer: ATCCCATCACCATCTTCCAG; GAPDH gene reverse primer: CCATCACGCCACAGTTTCC). Each assay was carried out on an Applied Biosystems 7300 Real-Time PCR system in triplicate and 
expression fold-changes were derived using the comparative $C_{t}$ method. Relative quantification was calculated using GAPDH as an endogenous control. Fold changes were calculated as the following Equations (1) and (2):

$$
\text { Fold change }=2^{-\Delta \Delta \mathrm{Ct}}
$$

$2^{-\Delta \Delta \mathrm{Ct}}=\left[\left(\mathrm{C}_{\mathrm{t}}\right.\right.$ gene of interest $-\mathrm{C}_{\mathrm{t}}$ internal control $)$ treated sample $-\left(\mathrm{C}_{\mathrm{t}}\right.$ gene of interest $-\mathrm{C}_{\mathrm{t}}$ internal control) untreated control)]

\subsection{Antioxidant Characteristics of Adlay Extract}

The antioxidant characteristics of adlay seed SFE was first determined by measuring the DPPH scavenging ability as previously described [27]. The ABTS decolorization assays were conducted as previously described. The $\mathrm{ABTS}^{+}$chromophore necessary for the assay was generated from a $7 \mathrm{mM}$ stock solution of ABTS by mixing with $2.45 \mathrm{mM}$ potassium persulfate solution and leaving the mixture in the dark for $6 \mathrm{~h}$ before use. One $\mathrm{mL}$ of the $\mathrm{ABTS}^{+}$thus formed was incubated with different concentrations of adlay extract for $10 \mathrm{~min}$ and the absorbance change was quantified at $734 \mathrm{~nm}$. The $\mathrm{ABTS}^{+}$scavenging capacity of adlay extract thus determined was compared with that of vitamin $\mathrm{C}$ $(50 \mu \mathrm{M})$ and butylated hydroxyanisole (BHA; $0.1 \mathrm{mg} / \mathrm{mL})[28]$.

The reducing capacity was determined according to previously published methods [29]. The test sample was mixed with phosphate buffer $(2.5 \mathrm{~mL}, 0.2 \mathrm{M}, \mathrm{pH} 6.6)$ and potassium ferricyanide $\left[\mathrm{K}_{3} \mathrm{Fe}(\mathrm{CN})_{6}\right](2.5 \mathrm{~mL}, 1 \% \mathrm{w} / \mathrm{v})$. The mixture was incubated at $50{ }^{\circ} \mathrm{C}$ for $20 \mathrm{~min}$. At the end of the reaction, $2.5 \mathrm{~mL}$ of trichloroacetic acid $(10 \% \mathrm{w} / \mathrm{v})$ was added to the mixture, and it was centrifuged at $1000 \times \mathrm{g}$ for $10 \mathrm{~min}$. The supernatant $(2.5 \mathrm{~mL})$ was treated with distilled water $(2.5 \mathrm{~mL})$ and $\mathrm{FeCl}_{3}$ $(0.5 \mathrm{~mL}, 0.1 \% \mathrm{w} / \mathrm{v})$, and the absorbance of the final solution was measured at $700 \mathrm{~nm}$ in a UV-Vis spectrophotometer. The content of total phenolics was determined with the Folin-Ciocalteu reagent [30] and gallic acid $(20 \mu \mathrm{g} / \mathrm{mL})$ was used as a positive standard. Different concentrations of samples were prepared in $80 \%$ of methanol. One-hundred microliters of sample were dissolved in $500 \mu \mathrm{L}$ (1/10 dilution) of the Folin-Ciocalteu reagent and $1 \mathrm{~mL}$ of distilled water. The solutions were mixed and incubated at room temperature. After $1 \mathrm{~min}, 1.5 \mathrm{~mL}$ of $20 \%$ sodium carbonate solution was added. The final mixture was shaken and then incubated for $2 \mathrm{~h}$ in the dark at room temperature. The absorbance of samples was measured at $760 \mathrm{~nm}$.

The cellular ROS levels were determined with the 2',7'-dichlorofluorescein diacetate (DCFH-DA) method [31]. B16F10 melanoma cells were cultured in 24-well plates $\left(5 \times 10^{4}\right.$ cells in $1 \mathrm{~mL}$ of DMEM medium) and treated with various concentrations of adlay extract for $24 \mathrm{~h}$. The cells were then incubated with $24 \mathrm{mM} \mathrm{H} \mathrm{O}_{2}$ at $37{ }^{\circ} \mathrm{C}$ for $30 \mathrm{~min}$. After incubation, DCFH-DA was added to the wells, and the cells were cultured for $30 \mathrm{~min}$. Following this treatment, the cells were washed with phosphate-buffered saline and trypsinized with trypsin/EDTA, and the fluorescence intensities of DCF were measured at excitation wavelength $504 \mathrm{~nm}$ and emission wavelength $524 \mathrm{~nm}$ using a fluorescent reader Fluoroskan Ascent (Thermo Scientific, Vantaa, Finland). The data were analyzed with Ascent software (Thermo Scientific, Vantaa, Finland). 


\subsection{Statistical Analysis}

Statistical analysis of the experimental data points was performed by the ANOVA test, which was used for comparison of measured data using SPSS 12.0 statistical software (SPSS INC. Chicago, IL, USA). Differences were considered to be statistically significant at $p<0.05$.

\section{Conclusions}

The current study constitutes the first report on the potential inhibitory effect of adlay extract on the melanin biosynthesis in B16 melanoma cells. Our studies also demonstrate the presence of powerful antioxidant activities in this extract. The inhibition of melanin production observed in the present study could be due a number of factors such as inhibition of tyrosinase, TRP-1, and TRP-2, the reduced expression of MITF, and due to depletion of cellular ROS. Based on our results, we forecast that adlay extract could be used as powerful skin lightening compound possessing antioxidant properties in the skin care industry.

\section{Acknowledgments}

This study was financially supported by the National Science Council, Taiwan, under Grant no. NSC 102-2221-E-241-019 and NSC 102-2632-B-241-001-MY3.

\section{Author Contributions}

Huey-Chun Huang conducted the tyrosinase-related studies, participated in the enzyme assays and drafted the manuscript. Wan-Yu Hsieh conducted the antioxidant experiments. Yu-Lin Niu extracted the adlay seeds and conducted western blotting. Tsong-Min Chang participated in design and coordination of the study, performed the statistical analysis and drafted the manuscript. All authors read and approved the final manuscript.

\section{Conflicts of Interest}

The authors declare no conflict of interest.

\section{References}

1. Garcia, A.; Fulton, J.E. The combination of glycolic acid and hydroquinone or kojic acid for the treatment of melasma and related conditions. Dermatol. Surg. 1996, 22, 443-447.

2. Virador, V.M.; Kobayashi, N.; Matsunaga, J.; Hearing, V.J. A standardized protocol for assessing regulators of pigmentation. Anal. Biochem. 1999, 270, 207-219.

3. Rendon, M.I.; Gaviria, J.I. Review of Skin-Lightening Agents. Dermatol. Surg. 2005, 31, 886-890.

4. Hearing, V.J.; Jiménez, M. Mammalian tyrosinase-The critical regulatory control point in melanocyte pigmentation. Int. J. Biochem. 1987, 19, 1141-1147.

5. Tsukamoto, K.; Jackson, I.J.; Urabe, K.; Montague, P.M.; Hearing, V. A second tyrosinase-related protein, TRP-2, is a melanogenic enzyme termed DOPAchrome tautomerase. EMBO J. 1992, 11, 519-526. 
6. Meyskens, F.L., Jr.; Chau, H.V.; Tohidian, N.; Buckmeier, J. Luminol-enhanced chemiluminescent response of human melanocytes and melanoma cells to hydrogen peroxide stress. Pigment Cell Res. 1997, 10, 184-189.

7. Funasaka, Y.; Komoto, M.; Ichihashi, M. Depigmenting effect of alpha-tocopheryl ferulate on normal human melanocytes. Pigment Cell Res. 2000, 13 (Suppl. 8), 170-174.

8. Chakraborty, A.K.; Funasaka, Y.; Slominski, A.; Ermak, G.; Hwang, J.; Pawelek, J.M.; Ichihashi, M. Production and release of proopiomelanocortin (POMC) derived peptides by human melanocytes and keratinocytes in culture: Regulation by ultraviolet B. BBA Mol. Cell Res. 1996, 1313, 130-138.

9. Sasaki, M.; Kizawa, K.; Igarashi, S.; Horikoshi, T.; Uchiwa, H.; Miyachi, Y. Suppression of melanogenesis by induction of endogenous intracellular metallothionein in human melanocytes. Exp. Dermatol. 2004, 13, 465-471.

10. Yamakoshi, J.; Otsuka, F.; Sano, A.; Tokutake, S.; Saito, M.; Kikuchi, M.; Kubota, Y. Lightening effect on ultraviolet-induced pigmentation of guinea pig skin by oral administration of a proanthocyanidin-rich extract from grape seeds. Pigment Cell Res. 2003, 16, 629-638.

11. Sies, H.; Stahl, W. Nutritional protection against skin damage from sunlight. Annu. Rev. Nutr. 2004, 24, 173-200.

12. Overwijk, W.W.; Restifo, N.P. B16 as a mouse model for human melanoma. Curr. Protoc. Immunol. 2001, doi:10.1002/0471142735.im2001s39.

13. Otsuka, H.; Hirai, Y.; Nagao, T.; Yamasaki, K. Anti-inflammatory activity of benzoxazinoids from roots of Coix lachryma-jobi var. ma-yuen. J. Nat. Prod. 1988, 51, 74-79.

14. Chen, H.-J.; Shih, C.-K.; Hsu, H.-Y.; Chiang, W. Mast cell-dependent allergic responses are inhibited by ethanolic extract of adlay (Coix lachryma-jobi L. var. ma-yuen Stapf) testa. J. Agric. Food Chem. 2010, 58, 2596-2601.

15. Seo, W.-G.; Pae, H.-O.; Chai, K.-Y.; Yun, Y.-G.; Kwon, T.-H.; Chung, H.-T. Inhibitory effects of methanol extract of seeds of job's tears (Coix Lachryma-Jobi L Var. Ma-Yuen) on nitric oxide and superoxide production in raw 264.7 macrophages. Immunopharm. Immunotoxicol. 2000, 22, 545-554.

16. Kuo, C.-C.; Shih, M.-C.; Kuo, Y.-H.; Chiang, W. Antagonism of free-radical-induced damage of adlay seed and its antiproliferative effect in human histolytic lymphoma U937 monocytic cells. J. Agric. Food Chem. 2001, 49, 1564-1570.

17. Busca, R.; Ballotti, R. Cyclic AMP a key messenger in the regulation of skin pigmentation. Pigment Cell Res. 2000, 13, 60-69.

18. Cheng, S.-L.; Liu, R.H.; Sheu, J.-N.; Chen, S.-T.; Sinchaikul, S.; Tsay, G.J. Toxicogenomics of A375 human malignant melanoma cells treated with arbutin. J. Biomed. Sci. 2007, 14, 87-105.

19. García-Gavín, J.; González-Vilas, D.; Fernández-Redondo, V.; Toribio, J. Pigmented contact dermatitis due to kojic acid. A paradoxical side effect of a skin lightener. Contact Dermat. 2010, 62, 63-64.

20. Emerit, I. Free radicals and aging of the skin. EXS 1992, 62, 328-341.

21. Sapkota, K.; Park, S.-E.; Kim, J.-E.; Kim, S.; Choi, H.-S.; Chun, H.-S.; Kim, S.-J. Antioxidant and antimelanogenic properties of chestnut flower extract. Biosci. Biotechnol. Biochem. 2010, 74, $1527-1533$. 
22. Ding, H.-Y.; Chou, T.-H.; Lin, R.-J.; Chan, L.-P.; Wang, G.-H.; Liang, C.-H. Antioxidant and antimelanogenic behaviors of Paeonia suffruticosa. Plant Food Hum. Nutr. 2011, 66, 275-284.

23. Bilodeau, M.L.; Greulich, J.D.; Hullinger, R.L.; Bertolotto, C.; Ballotti, R.; Andrisani, O.M. BMP-2 stimulates tyrosinase gene expression and melanogenesis in differentiated melanocytes. Pigment Cell Res. 2001, 14, 328-336.

24. Tsuboi, T.; Kondoh, H.; Hiratsuka, J.; Mishima, Y. Enhanced melanogenesis induced by tyrosinase gene-transfer increases boron-uptake and killing effect of boron neutron capture therapy for amelanotic melanoma. Pigment Cell Res. 1998, 11, 275-282.

25. Yang, J.Y.; Koo, J.H.; Song, Y.G.; Kwon, K.B.; Lee, J.H.; Sohn, H.S.; Park, B.H.; Jhee, E.C.; Park, J.W. Stimulation of melanogenesis by scoparone in B16 melanoma cells. Acta Pharmacol. Sin. 2006, 27, 1467-1473.

26. Huang, H.-C.; Chang, T.-M.; Chang, Y.-J.; Wen, H.-Y. UVB Irradiation Regulates ERK1/2- and p53-Dependent Thrombomodulin Expression in Human Keratinocytes. PLoS One 2013, 8, e67632.

27. Brand-Williams, W.; Cuvelier, M.E.; Berset, C. Use of a free radical method to evaluate antioxidant activity. Food Sci. Technol. 1995, 28, 25-30.

28. Re, R.; Pellegrini, N.; Proteggente, A.; Pannala, A.; Yang, M.; Rice-Evans, C. Antioxidant activity applying an improved ABTS radical cation decolorization assay. Free Radic. Biol. Med. 1999, 26, 1231-1237.

29. Oyaizu, M. Studies on products of browing reaction: Antioxidative activity of product of browing reaction preapared from glucosamine. Jpn. J. Nutr. 1986, 44, 307-315.

30. Galato, D.; Ckless, K.; Susin, M.F.; Giacomelli, C.; Ribeiro-do-Valle, R.M.; Spinelli, A. Antioxidant capacity of phenolic and related compounds: Correlation among electrochemical, visible spectroscopy methods and structure-antioxidant activity. Redox Rep. 2001, 6, 243-250.

31. Murrant, C.L.; Reid, M.B. Detection of reactive oxygen and reactive nitrogen species in skeletal muscle. Microsc. Res. Tech. 2001, 55, 236-248.

(C) 2014 by the authors; licensee MDPI, Basel, Switzerland. This article is an open access article distributed under the terms and conditions of the Creative Commons Attribution license (http://creativecommons.org/licenses/by/3.0/). 